\title{
Evolution of Cooperation and Coordination in a Dynamically Networked Society
}

\author{
Enea Pestelacci \\ Marco Tomassini \\ Leslie Luthi \\ Information Systems Department \\ University of Lausanne \\ Switzerland \\ enea.pestelacci@unil.ch \\ Marco.Tomassini@unil.ch \\ 1luthi@gmail.com
}

\begin{abstract}
Situations of conflict giving rise to social dilemmas are widespread in society and game theory is one major way in which they can be investigated. Starting from the observation that individuals in society interact through networks of acquaintances, we model the co-evolution of the agents' strategies and of the social network itself using two prototypical games, the Prisoner's Dilemma and the Stag-Hunt. Allowing agents to dismiss ties and establish new ones, we find that cooperation and coordination can be achieved through the self-organization of the social network, a result that is nontrivial, especially in the Prisoner's Dilemma case. The evolution and stability of cooperation implies the condensation of agents exploiting particular game strategies into strong and stable clusters which are more densely connected, even in the more difficult case of the Prisoner's Dilemma.
\end{abstract}

\section{Keywords}

co-evolving networks, community structure, evolution of cooperation, social networks 
In this article we study the behavior of a population of agents playing some simple two-person, one-shot noncooperative game. Game theory (Meyerson 1991) deals with social interactions where two or more individuals take decisions that will mutually influence each other. It is thus a view of collective systems in which global social outcomes emerge as a result of the interaction of the individual decisions made by each agent. Some extremely simple games lead to puzzles and dilemmas that have a deep social meaning. The most widely known among these games is the Prisoner's Dilemma (PD), a universal metaphor for the tension that exists between social welfare and individual selfishness. It stipulates that, in situations where individuals may either cooperate or defect, they will rationally choose the latter. However, cooperation would be the preferred outcome when global welfare is considered. Other simple games that give rise to social dilemmas are the Hawk-Dove and the Stag-Hunt ( $\mathrm{SH}$ ) games.

In practice, however, cooperation and coordination on common objectives is often seen in human and animal societies (Axelrod 1984; Skyrms 2004). Coordinated behavior, such as having both players cooperating in the $\mathrm{SH}$, is a bit less problematic as this outcome, being a Nash equilibrium (as explained below), is not ruled out by theory. For the PD, in which cooperation is theoretically doomed between rational agents, several mechanisms have been invoked to explain the emergence of cooperative behavior. Among them, repeated interaction, reputation, and belonging to a recognizable group have often been mentioned (Axelrod 1984). Yet, Nowak and May (1992) showed that the simple fact that players are arranged according to a spatial structure and only interact with neighbors is sufficient to sustain a certain amount of cooperation even when the game is played anonymously and without repetition. Nowak and May's study and much of the following work were based on regular structures such as two-dimensional grids (see Nowak and Sigmund 2000 for a recent review). Nevertheless, many actual social networks usually have a topological structure that is neither regular nor random but rather of the small-world type. Roughly speaking, small-world networks are graphs in which any node is relatively close to any other node. In this sense, they are similar to random graphs but unlike regular lattices. However, in contrast to random graphs, they also have a certain amount of local structure, as measured, for instance, by a quantity called the clustering coefficient, which essentially represents the probability that two neighbors of a given node are themselves connected (Newman 2003 offers an excellent review of the subject). Some work has been done in recent years in the direction of using those more realistic networks, including actual social networks. In particular we mention Santos and Pacheco's (2005) work on scale-free networks, work on Watts-Strogatz small-world graphs (Abramson and Kuperman 2001; Tomassini et al. 2006), and work on model and real social networks (Luthi et al. 2008). A recent contribution focuses on repeated games and learning (Wang et al. 2008); Szabó and Fáth (2007) have published an excellent and complete review of work done up to 2006. These investigations have convincingly shown that a realistic structure of society, with interactions mainly limited to neighbors in the network, is sufficient in allowing cooperative and coordinated behavior to emerge without making any particular assumptions about the rationality of the actors or their computational and forecasting capabilities.

Most of the aforementioned studies have assumed a fixed population size and structure, which amounts to dealing with a closed system and ignoring any fluctuations in the system's size and internal interactions. However, real social networks, such as friendship or collaboration networks, are not in an equilibrium state, but are open systems that continually evolve with new agents joining or leaving the network, and relationships (i.e., links in network terms) being made or dismissed by agents already in the network (Barabási et al. 2002; Kossinets and Watts 2006; Tomassini and Luthi 2007). The motivation for the present work is to reintroduce these coupled dynamics into our model and to investigate under which conditions, if any, cooperative and coordinated behavior may emerge and be stable. In this article, we shall deal with networked populations in which the number of players remains constant whereas the interaction structure, i.e., who interacts with whom, does not stay fixed; on the contrary, it changes in time, and its variation is dictated by the very games that are being played by the agents. A related goal of the present work is to study the topological structures of the emergent networks and their relationships with the strategic choices of the agents.

Some previous research has been done on evolutionary games on dynamic networks (Skyrms and Pemantle 2000; Zimmermann and Eguíluz 2005; Luthi et al. 2006; Santos et al. 2006). Skyrms and Pemantle (2000) was recently brought to our attention by a reviewer. It is one of the first important attempts to study the kind of networks that form under a given game and, as such, is closely related to the work we describe here. The main ideas are similar to ours: agents start interacting at random according to some game's payoff matrix and, as they evolve their game strategy according to their observed payoffs, they also have a chance of breaking ties and forming new ones, thus giving rise to a social network. The main differences with the present work is that the number of agents Skyrms and Pemantle used is low, of the order of 10 instead of the $10^{3}$ used here. This allows us to study the topological and statistical nature of the evolving networks in a way that is not possible with a few agents, while Skyrms's and Pemantle's work is more quantitative in the study of the effects of the stochastic dynamics on the strategy and network evolution process. The work of Zimmermann and Eguíluz (2005) is based on similar considerations: A rather large population initially has a random structure. Agents in the population play the one-shot, two-person 
PD game against each other and change their strategy by copying the strategy of the more successful agent in their neighborhood. They also have the possibility of dismissing interactions between defectors and of rewiring them randomly in the population. The main differences with the present work are the following. Instead of just considering symmetrical undirected links, we have a concept of two directed, weighted links between pairs of agents. In our model there is a finite probability of breaking any link, not only links between defectors, although defector-defector and cooperator-defector links are much more likely to be dismissed than cooperatorcooperator links. When a link is broken it is rewired randomly in Zimmermann and Eguíluz's (2005) model, while we use a link redirection process favoring neighbors with respect to more relationally distant agents. Zimmermann and Eguíluz only study the PD using a reduced parameter space. We study both the PD and the SH games covering a much larger parameter space. Concerning timing of events, we use an asynchronous update policy for the agents' strategies, while update is synchronous in Zimmermann and Eguíluz (2005). Finally, instead of a best-takes-over discrete rule, we use a smoother strategy update rule, which changes an agent's strategy with a probability proportional to the payoff difference. Santos et al. (2006) deal with similar issues in a more recent paper. However, they use a different algorithm for severing an undirected link between the two agents which, again, does not include the concept of a link weight. Furthermore, the SH game is only mentioned in passing, and their strategy update rule is different. In particular, they do not analyze in detail the statistical structure of the emerging networks, as we do here. Other differences with the aforementioned related works will be described in the discussion and analysis of results. Finally, our own previous work (Luthi et al. 2006) also deals with the co-evolution of strategy and structure in an initially random network. However, it is very different from the one presented here since we there used a semi-rational threshold decision rule for a family of games similar to, but not identical to, the PD. Furthermore, the idea of a bidirectional weighted link between agents was absent, and link rewiring was random.

This article is structured as follows. In the next section, we give a brief description of the games used in our study. This part is intended to make the article self-contained. We then present a detailed description of our model of co-evolving dynamical networks and discuss the simulation results and their significance for the social networks. In the final section, we offer our conclusions and discuss possible extensions and future work.

\section{Social Dilemmas}

The two representative games studied here are the PD and the $\mathrm{SH}$, whose significance and main results we briefly summarize. More detailed accounts can be found elsewhere, for instance in Axelrod (1984) and Skyrms (2004). In their simplest form, they are two-person, two-strategy, symmetric games with the following payoff bi-matrix:

\begin{tabular}{l|ll} 
& $\mathrm{C}$ & $\mathrm{D}$ \\
\hline $\mathrm{C}$ & $(R, R)$ & $(S, T)$ \\
$\mathrm{D}$ & $(T, S)$ & $(P, P)$
\end{tabular}

In this matrix, $R$ stands for the reward the two players receive if they both cooperate (C), $P$ is the punishment for bilateral defection (D), and $T$ is the temptation, i.e., the payoff a player receives if it defects while the other player cooperates. In this case, the cooperator gets the sucker's payoff, $S$. In both games, the condition $2 R>T+S$ is imposed so that mutual cooperation is preferred over an equal probability of unilateral cooperation and defection. For the PD, the payoff values are ordered numerically in the following way: $T>R>P>S$. Defection is always the best rational individual choice in the $\mathrm{PD}$; (D, D) is the unique Nash equilibrium (NE) and also an evolutionarily stable strategy (ESS) (Myerson 1991; Weibull 1995). Mutual cooperation would be preferable but it is a strongly dominated strategy.

In the $\mathrm{SH}$, the ordering is $R>T>P>S$, which means that mutual cooperation $(\mathrm{C}, \mathrm{C})$ is the best outcome, Paretosuperior, and an NE. An outcome of a game is Pareto-superior when there is no outcome that will make all players better off. However, there is a second equilibrium in which both players defect $(\mathrm{D}, \mathrm{D})$ and which is somewhat "inferior" to the previous one, although perfectly equivalent from a NE point of view. The (D, D) equilibrium is less satisfactory, yet "risk-dominant," since playing it "safe" by choosing strategy D guarantees at least a payoff of $P$, while playing $\mathrm{C}$ might expose a player to a $\mathrm{D}$ response by her opponent, with the ensuing minimum payoff $S$. Here the dilemma is represented by the fact that the socially preferable coordinated equilibrium $(\mathrm{C}, \mathrm{C})$ might be missed for "fear" that the other player will play $D$ instead. There is a third mixed-strategy NE in the game, but it is commonly dismissed because of its inefficiency and also because it is not an ESS (Weibull 1995). Although the PD has received much more attention in the literature than the $\mathrm{SH}$, the latter is also very useful, especially as a metaphor of coordinated social behavior for mutual benefit. These aspects are nicely explained in Skyrms (2004).

\section{Model Description}

Our model is strictly local as no player uses information other than the one concerning itself and the players it is directly connected to. In particular, each agent knows its own current strategy and payoff, and the current strategies and payoffs of its immediate neighbors. Moreover, as the model is an evolutionary one, no rationality - in the sense of game theory-is needed (Weibull 1995). Players just adapt their behavior such 


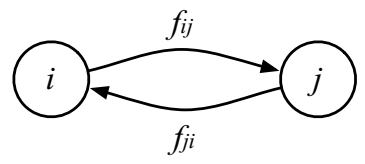

Figure 1.

Schematic representation of mutual trust between two agents through the strengths of their links.

that they copy more successful strategies in their environment with higher probability, a process commonly called imitation in the literature (Hofbauer and Sigmund 1998). Furthermore, they are able to locally assess the worth of an interaction and possibly dismiss a relationship that does not pay off enough. The model and its dynamics are described in detail in the following sections.

\section{Network and Interaction Structure}

The network of agents will be represented as an undirected graph $G(V, E)$, where the set of vertices $V$ represents the agents, while the set of edges (or links) $E$ represents their symmetric interactions. The population size $N$ is the cardinality of $V$. A neighbor of an agent $i$ is any other agent $j$ such that there is an edge $\{i j\} \in E$. The set of neighbors of $i$ is called $V_{i}$ and its cardinality is the degree $k_{i}$ of vertex $i \in V$. The average degree of the network will be called $\bar{k}$.

Although from the network structure point of view there is a single undirected link between a player $i$ and another player $j \in V_{i}$, we shall maintain two links: one going from $i$ to $j$ and another one in the reverse direction (see Figure 1). Each link has a weight or "force" $f_{i j}$ (respectively $f_{j i}$ ). This weight, say $f_{i j}$, represents in an indirect way an abstract quality that could be related to the "trust" player $i$ attributes to player $j$; it may take any value in $[0,1]$ and its variation is dictated by the payoff earned by $i$ in each encounter with $j$, as explained below.

We point out that we do not believe that this model could represent, however roughly, a situation of genetic relatedness in a human or animal society. In this case, at the very least, at the outset link strengths between close relatives should be higher than the average forces in the whole network, and such groups should form cliques of completely connected agents. In contrast, we start our simulations from random relationships and a constant average link strength (see below). Thus, our simplified model is closer to one in which relationships between agents are only of socioeconomic nature.

The idea behind the introduction of the forces $f_{i j}$ is loosely inspired by the potentiation/depotentiation of connections between neural networks, an effect known as the Hebb rule (Hebb 1949). In our context, it can be seen as a kind of "memory" of previous encounters. However, it must be distinguished from the memory used in iterated games, in which players "remember" a certain amount of previous moves and can thus conform their future strategy on the analysis of those past encounters (Myerson 1991). Our interactions are strictly one-shot, i.e., players "forget" the results of previous rounds and cannot recognize previous partners and their possible playing patterns. However, a certain amount of past history is implicitly contained in the numbers $f_{i j}$ and this information may be used by an agent when it will come to decide whether or not an interaction should be dismissed (see below). ${ }^{1}$ This bilateral view of a relationship is, to our knowledge, new in evolutionary game models on graphs.

We also define a quantity $s_{i}$, called satisfaction of an agent $i$, which is the sum of all the weights of the links between $i$ and its neighbors $V_{i}$ divided by the total number of links $k_{i}$ :

$s_{i}=\frac{\sum j \in V_{i} f_{i j}}{k_{i}}$.

We clearly have $0 \leq s_{i} \leq 1$.

\section{Initialization}

The constant size of the network during the simulations is $N=1000$. The initial graph is generated randomly with a mean degree comprised between $\bar{k}=5$ and $\bar{k}=20$. These values of $\bar{k}$ are of the order of those actually found in many social networks (see, for instance, Newman 2001; Barabási et al. 2002; Kossinets and Watts 2006; Tomassini et al. 2007). Players are distributed uniformly at random over the graph vertices with 50\% cooperators. Forces between any pair of neighboring players are initialized at 0.5 . With $\bar{k}>1$ a random graph finds itself past the percolation phase transition (Bollobás 1998) and thus it has a giant connected component of size $O(N)$ while all the other components are of size $O(\log (N))$. We do not assume that the whole graph is connected, as isolated nodes will draw a random link during the dynamics (see below).

Before starting the simulations, another parameter, $q$, has to be set. This is akin to a "temperature" or noise level; $q$ is a real number in $[0,1]$ and it represents the frequency with which an agent wishes to dismiss a link with one of its neighbors. The higher $q$, the faster the link reorganization in the network. This parameter has a role analogous to Zimmermann and Eguíluz's (2005) "plasticity"; it controls the speed at which topological changes occur in the network. As social networks may structurally evolve at widely different speeds, depending on the kind of interaction between agents, this factor might play a role in the model. For example, e-mail networks change their structure at a faster pace than, say, scientific collaboration networks (Kossinets and Watts 2006; Tomassini and Luthi 2007). A similar coupling of time scales between strategy update and topological update also occurs in Skyrms and Pemantle (2000) and Santos et al. (2006).

\section{Timing of Events}

Usually, agents systems such as the present one are updated synchronously, especially in evolutionary game theory simulations (Nowak and May 1992; Santos and Pacheco 2005; 
Zimmermann and Eguíluz 2005; Luthi et al. 2008). However, there are doubts about the physical signification of simultaneous update (Huberman and Glance 1993). For one thing, it is physically unfeasible, strictly speaking, as it would require a global clock, while real extended systems in biology and society in general have to take into account finite signal propagation speed. Furthermore, simultaneity may cause some artificial effects in the dynamics that are not observed in real systems (Huberman and Glance 1993; Luthi et al. 2006). Fully asynchronous update, i.e., updating a randomly chosen agent at a time with or without replacement also seems a rather arbitrary extreme case that is not likely to represent reality very accurately. In view of these considerations, we have chosen to update our population in a partially synchronous manner. In practice, we define a fraction $f=n / N$ (with $N=a n, a \in N$ ) and, at each simulated discrete time step, we update only $n \leq N$ agents randomly chosen with replacement. This is called a microstep. After $N / n$ microsteps, called a macrostep, $N$ agents will have been updated, i.e., the whole population will have been updated in the average. With $n=N$ we recover the fully synchronous update, while $n=1$ gives the extreme case of the fully asynchronous update. Varying $f$ thus allows one to investigate the role of the update policy on the dynamics. We study several different values of $f$, but we mainly focus on $f=0.01$.

\section{Strategy and Link Dynamics}

Here we describe in detail how individual strategies, links, and link weights are updated. Once a given node $i$ is chosen to be activated, i.e., belongs to the fraction $f$ of nodes that are to be updated in a given microstep, $i$ goes through the following steps:

- If the degree of agent $i, k_{i}=0$ then player $i$ is an isolated node. In this case a link with strength 0.5 is created from $i$ to a player $j$ chosen uniformly at random among the other $N-1$ players in the network.

- Otherwise,

- either agent $i$ updates its strategy according to a local replicator dynamics rule with probability $1-q$ or, with probability $q$, agent $i$ may delete a link with a given neighbor $j$ and creates a new 0.5 force link with another node $k$;

- the forces between $i$ and its neighbors $V_{i}$ are updated.

Let us now describe each step in some more detail.

Strategy evolution We use a local version of replicator dynamics (RD) as described in Hauert and Doebeli (2004) and modified in Luthi et al. (2008) to take into account the fact that the number of neighbors in a degree-inhomogeneous network can be different for different agents. The local dynamics of a player $i$ only depends on its own strategy and on the strategies of the $k_{i}$ players in its neighborhood $V_{i}$. Let us call $\pi_{i j}$ the payoff player $i$ receives when interacting with neighbor $j$. This payoff is defined as

$$
\pi_{i j}=\sigma_{i}(t) M \sigma_{j}^{T}(t)
$$

where $M$ is the payoff matrix of the game (see the next section) and $\sigma_{i}(t)$ and $\sigma_{j}(t)$ are the strategies played by $i$ and $j$ at time $t$. The quantity

$$
\hat{\Pi}_{i}(t)=\sum_{j \in V_{i}} \pi_{i j}(t)
$$

is the accumulated payoff collected by player $i$ at time step $t$. The rule according to which agents update their strategies is the conventional RD in which strategies that do better than the average increase their share in the population, while those that fare worse than average decrease. To update the strategy of player $i$, another player $j$ is drawn at random from the neighborhood $V_{i}$. It is assumed that the probability of switching strategy is a function $\phi$ of the payoff difference, where $\phi$ is a monotonically increasing function (Hofbauer and Sigmund 1998). Strategy $\sigma_{i}$ is replaced by $\sigma_{j}$ with probability

$$
p_{i}=\phi\left(\hat{\Pi}_{j}-\hat{\Pi}_{i}\right)
$$

The major differences with standard RD is that two-person encounters between players are only possible among neighbors, instead of being drawn from the whole population, and the latter is finite in our case. Other commonly used strategy update rules include imitating the best in the neighborhood (Nowak and May 1992; Zimmermann and Eguíluz 2005), or replicating in proportion to the payoff (Hauert and Doebeli 2004; Tomassini et al. 2006). Although these rules are acceptable alternatives, they do not lead to RD and will not be dealt with here. We also note that the straight accumulated payoff $\hat{\Pi}_{i}$ has a technical problem when used on degree-inhomogeneous systems such as those studied here, where agents (i.e., nodes) in the network may have different numbers of neighbors. In fact, in this case $\hat{\Pi}_{i}$ does not induce invariance of the RD with respect to affine transformations of the game's payoff matrix as it should (Weibull 1995), and makes the results depend on the particular payoff values. Thus, we shall use a modified accumulated payoff $\Pi$ instead as defined in Luthi et al. (2008). This payoff, which is the standard accumulated payoff corrected with a factor that takes into account the variable number of neighbors an agent may have, does not suffer from the standard accumulated payoff limitations. 
Link evolution The active agent $i$, which has $k_{i} \neq 0$ neighbors, will, with probability $q$, attempt to dismiss an interaction with one of its neighbors. This is done in the following way. Player $i$ will look at its satisfaction $s_{i}$. The higher $s_{i}$, the more satisfied the player, since a high satisfaction is a consequence of successful strategic interactions with the neighbors. Thus, there should be a natural tendency to try to dismiss a link when $s_{i}$ is low. This is simulated by drawing a uniform pseudo-random number $r \in[0,1]$ and breaking a link when $r \geq s_{i}$. Assuming that the decision is taken to cut a link, which one, among the possible $k_{i}$, should be chosen? Our solution again relies on the strength of the relevant links. First a neighbor $j$ is chosen with probability proportional to $1-f_{i j}$, i.e., the stronger the link, the less likely it will be chosen. This intuitively corresponds to $i$ 's observation that it is preferable to dismiss an interaction with a neighbor $j$ that has contributed little to $i$ 's payoff over several rounds of play. However, in our system dismissing a link is not free: $j$ may "object" to the decision. The intuitive idea is that, in real social situations, it is seldom possible to take unilateral decisions: often there is a cost associated, and we represent this hidden cost by a probability $1-\left(f_{i j}+f_{j i}\right) / 2$ with which $j$ may refuse to be cut away. In other words, the link is less likely to be deleted if $j$ appreciates $i$, i.e., when $f_{i j}$ is high. A simpler solution would be to try to cut the weakest link, which is what happens most of the time anyway. However, with a finite probability of cutting any link, our model introduces a small amount of noise in the process which can be considered like "trembles" or errors in game theory (Myerson 1991) and which roughly reproduces decisions under uncertainty in the real world.

Assuming that the $\{i j\}$ link is finally cut, how is a new link to be formed? The solution adopted here is inspired by the observation that, in social networks, links are usually created more easily between people who have a mutual acquaintance than those who do not. First, a neighbor $k$ is chosen in $V_{i} \backslash\{j\}$ with probability proportional to $f_{i k}$, thus favoring neighbors $i$ trusts. Next, $k$ in turn chooses player $l$ in his neighborhood $V_{k}$ using the same principle, i.e., with probability proportional to $f_{k l}$. If $i$ and $l$ are not connected, a link $\{i l\}$ is created, otherwise the process is repeated in $V_{l}$. Again, if the selected node, say $m$, is not connected to $i$, a new link $\{i m\}$ is established. If this also fails, a new link between $i$ and a randomly chosen node is created. In all cases the new link is initialized with a strength of 0.5 in both directions. This rewiring process is schematically depicted in Figure 2 for the case in which a link can be successfully established between players $i$ and $l$ thanks to their mutual acquaintance $k$.

At this point, we would like to stress several important differences with previous work in which links can be dismissed in evolutionary games on networks (Zimmermann and Eguíluz 2005; Luthi et al. 2006; Santos et al. 2006). In Zimmermann

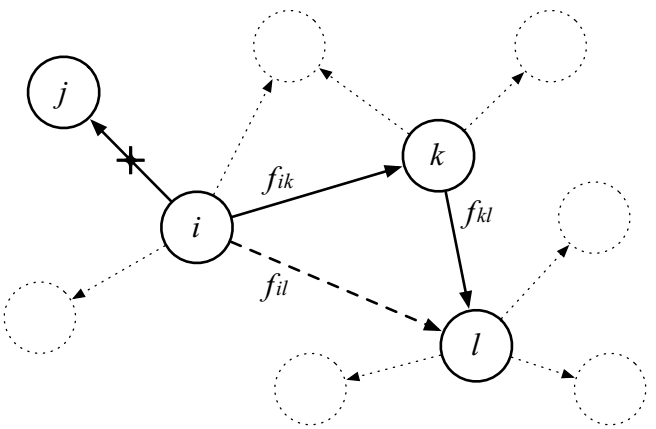

Figure 2.

Illustration of the rewiring of link $\{i j\}$ to $\{i l\}$. Agent $k$ is chosen to introduce player $l$ to $i$ (see text).

and Eguíluz's (2005) model, only links between defectors are allowed to be cut unilaterally and the study is restricted to the PD. Instead, in our case, any link has a finite probability to be abandoned, even a profitable link between cooperators if it is recent, although links that are more stable, i.e., have high strengths, are less likely to be rewired. This smoother situation is made possible thanks to our bilateral view of a link, which is completely different from the undirected choice made in Zimmermann and Eguíluz (2005).

In Santos et al.'s (2006) model, links can be cut by an unsatisfied player, where the concept of satisfaction is different from ours, and simply means that a cooperator or a defector will wish to break a link with a defector. The cut will be done with a certain probability that depends on the strategies of the two agents involved and their respective payoffs. Once a link between $i$ and $j$ is actually cut and, among the two players, $i$ is the one selected to maintain the link, the link is rewired to a random neighbor of $j$. If both $i$ and $j$ wish to cease their interaction, the link is attributed to $i$ or $j$ probabilistically, as a function of the respective payoffs of $i$ and $j$, and rewiring takes place from there. Thus, although both $i$ 's and $j$ 's payoffs are taken into consideration in the latter case, there is no analogon of our "negotiation" process as the concept of link strength is absent. In Luthi et al.'s (2006) model, links are cut according to a threshold decision rule and are rewired randomly anywhere in the network.

A final observation concerns the evolution of $\bar{k}$ in the network. While in Zimmermann and Eguíluz's (2005) and Santos et al.'s (2006) models the initial mean degree is strictly maintained during network evolution through the rewiring process, here it may increase slightly owing to the existence of isolated agents which, when chosen to be updated, will create a new link with another random agent. While this effect is of minor importance and only causes small fluctuations of $\bar{k}$, we point out that in real evolving networks the mean connectivity fluctuates too (Barabási et al. 2002; Kossinets and Watts 2006; Tomassini and Luthi 2007). 
Updating the link strengths Once the chosen agents have gone through their strategy or link update steps, the strengths of the links are updated accordingly in the following way:

$$
f_{i j}(t+1)=f_{i j}(t)+\frac{\pi_{i j}-\bar{\pi}_{i j}}{k_{i}\left(\pi_{\max }-\pi_{\min }\right)},
$$

where $\pi_{i j}$ is the payoff of $i$ when interacting with $j, \bar{\pi}_{i j}$ is the payoff earned by $i$ playing with $j$, if $j$ were to play his other strategy, and $\pi_{\max }\left(\pi_{\min }\right)$ is the maximal (minimal) possible payoff obtainable in a single interaction. This update is performed in both directions, i.e., both $f_{i j}$ and $f_{j i}$ are updated $\forall j \in V_{i}$ because both $i$ and $j$ get a payoff out of their encounter.

The following algorithms schematically describe the whole co-evolution process for one microstep:

Algorithm 1: Simulation of a Microstep of the Population Evolution

Data: $V$ is a set of players; $I$ is a subset of $n$ randomly selected players in $V ; V_{i}$ is the set of first neighbors of player $i ; f_{i j}$ is the strength of the oriented $\operatorname{arc}\{\overrightarrow{i j}\} ; r$ is a pseudo-random number $\in[0,1)$

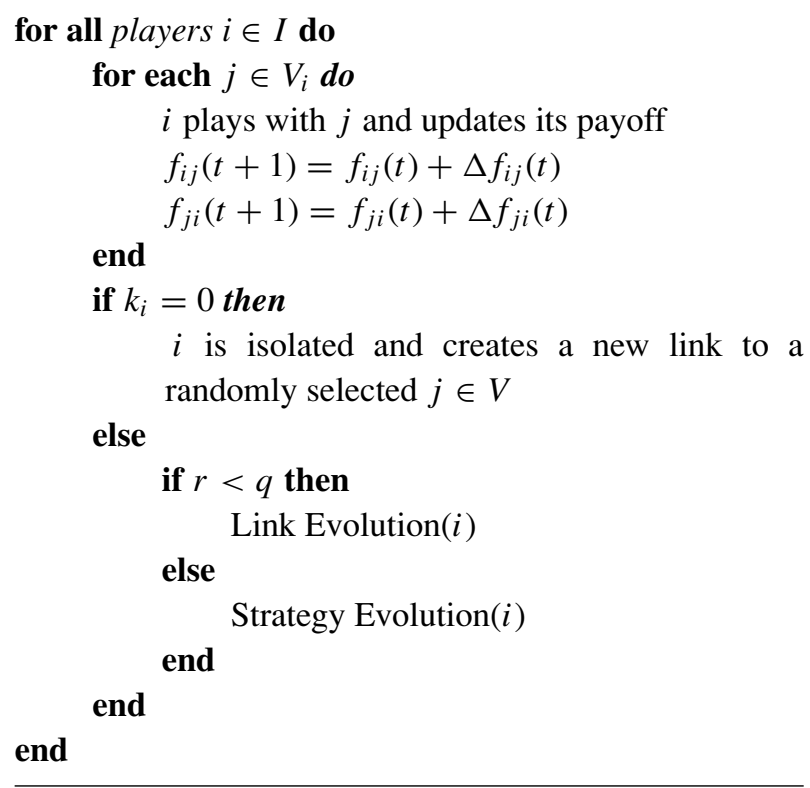

Algorithm 2: Link Evolution (i)

Data: $r_{1}$ and $r_{2}$ are pseudo-random numbers $\in[0,1) ; s_{i}$ is the satisfaction of player $i$

If $r_{l}>s_{i}$ then

$j \in V_{i}$ selected proportionally to $1-f_{i j}$

if $r_{2}>\left(f_{i j}+f_{j i}\right) / 2$ then

remove the link $\{i j\}$

$k \in V_{i}$ selected proportionally to $f_{i k}$

$l \in V_{k}$ selected proportionally to $f_{k l}$

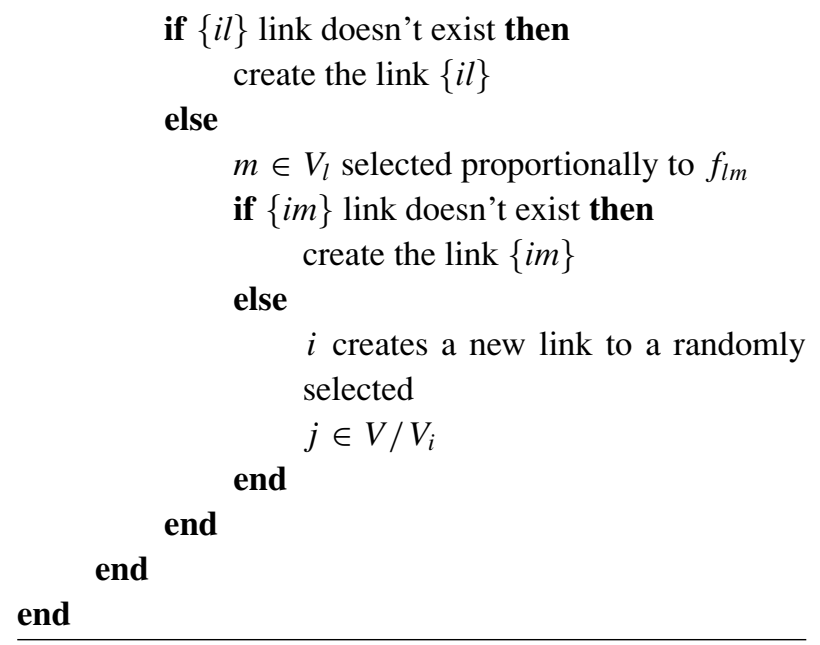

Algorithm 3: Strategy Evolution (i)

Data: $r$ is a pseudo-random number $\in[0,1) ; \Pi_{k}$ is the aggregated payoff of player $k ; \phi$ is a monotonically increasing function

$j \in V_{i}$ randomly selected

$p_{i}=\phi\left(\Pi_{j}-\Pi_{i}\right)$

if $r>p_{i}$ then

$i$ imitate the strategy of $j$

end

\section{Simulation Results}

\section{Simulation Parameters}

We simulate on our networks the two games previously mentioned in the opening section. For each game, we can explore the entire game space by limiting our study to the variation of only two parameters per game. This is possible without loss of generality owing to the invariance of Nash equilibria and $\mathrm{RD}$ under positive affine transformations of the payoff matrix using our payoff scheme (Weibull 1995). In the case of the PD, we set $R=1$ and $S=0$, and vary $1 \leq T \leq 2$ and $0 \leq P \leq 1$. For the SH, we decided to fix $R=1$ and $S=0$ and vary $0 \leq T \leq 1$ and $0 \leq P \leq T$. The reason we choose to set $T$ and $S$ in both the PD and the SH is to simply provide natural bounds on the values to explore for the remaining two parameters. In the PD case, $P$ is limited between $R=1$ and $S=0$ in order to respect the ordering of the payoffs $(T>R>P>S)$ and $T$ 's upper bound is equal to 2 due to the $2 R>T+S$ constraint. Had we fixed $R=1$ and $P=0$ instead, $T$ could be as big as desired, provided $S \leq 0$ is small enough. In the SH, setting $R=1$ and $S=0$ determines the range of $T$ and $P$ (since this time $R>T>P>S$ ). Note however, that for this game the only valid value pairs of $(T, P)$ are those that satisfy the $T>P$ constraint.

As stated in the opening section, we used networks of size $N=1000$, randomly generated with an average degree 

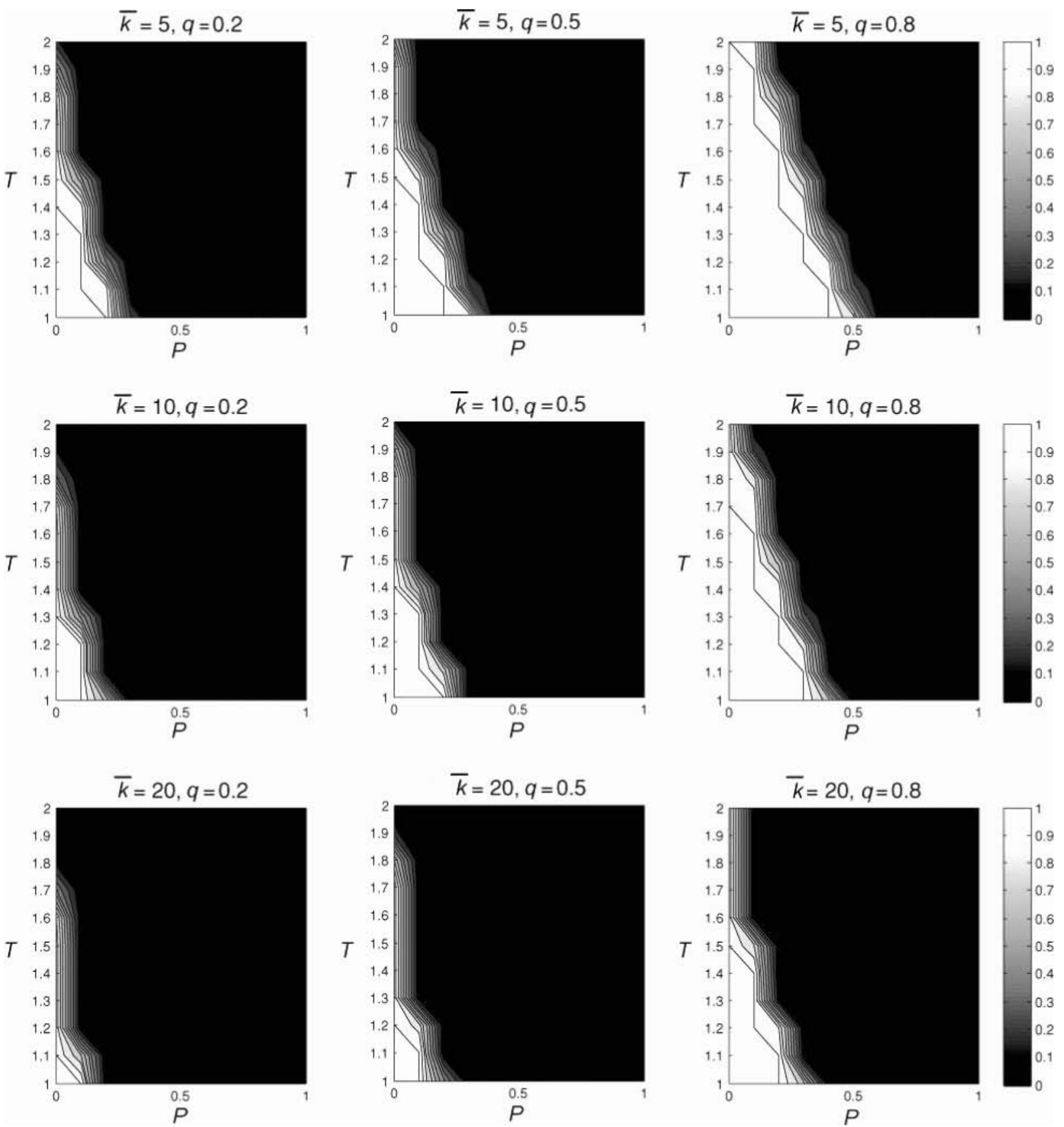

Figure 3.

Cooperation level for the PD in the game's configuration space. Darker gray means more defection.

$\bar{k} \in\{5,10,20\}$ and randomly initialized with $50 \%$ cooperators and $50 \%$ defectors. In all cases, the parameters are varied between their two bounds in steps of 0.1. For each set of values, we carry out 50 runs of at most 20,000 macrosteps each, using a fresh graph realization in each run. A run is stopped when all agents are using the same strategy, in order to be able to measure statistics for the population and for the structural parameters of the graphs. The system is considered to have reached a pseudo-equilibrium strategy state when the strategy of the agents (C or D) does not change over 150 further macrosteps, which means $15 \times 10$ individual updates.
We speak of pseudo-equilibria or steady states and not of true evolutionary equilibria because, as we shall see below, the system never quite reaches a totally stable state in the dynamical systems sense in our simulations but only transient states that persist for a long time.

\section{Cooperation and Stability}

Cooperation results for the PD in contour plot form are shown in Figure 3. We remark that, as observed in other structured populations, cooperation may thrive in a small but nonnegligible part of the parameter space. Thus, the added degree of 

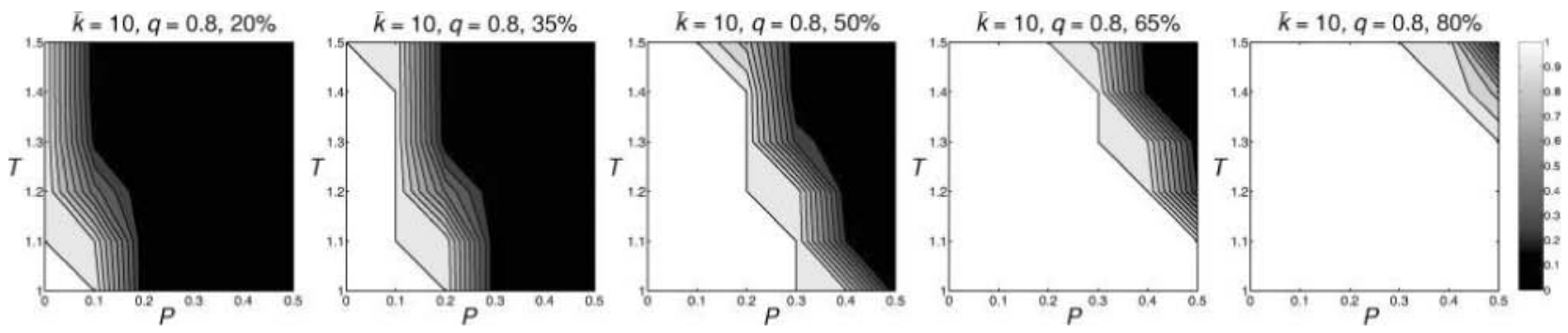

Figure 4.

Cooperation level for the PD starting with different fractions of cooperators increasing from $20 \%$ to $80 \%$ from left to right. Only the lower left quarter of the parameter space is shown. Results are the average of 50 independent runs.

freedom represented by the possibility of refusing a partner and choosing a new one does indeed help to find player's arrangements that help cooperation. This finding is in line with the results of Santos et al. (2006) and Zimmermann and Eguíluz (2005). Furthermore, the fact that our artificial society model differs from the latter two in several important ways also shows that the result is a rather robust one. When considering the dependence on the fluidity parameter $q$, one sees in Figure 3 that the higher $q$, the higher the cooperation level. This was expected since being able to break ties more often clearly gives cooperators more possibilities for finding and keeping fellow cooperators to interact with. This effect has been previously observed also in the works of the aforementioned authors and, as such, seems to be a robust finding, relatively independent of the other details of the models. The third parameter considered in Figure 3 is the mean degree $\bar{k}$. For a given value of $q$, cooperation becomes weaker as $\bar{k}$ increases. We believe that, as far as $\bar{k}$ is concerned, a realistic average characterization of actual social networks is represented by $\bar{k}=10$ (middle row in Figure 3) as seen, for instance, in the simulations of Newman (2001), Barabási et al. (2002), Kossinets and Watts (2006), and Tomassini et al. (2007). Higher average degrees do exist, but they are found either in web-based pseudo-social networks or in fairly special collaboration networks like the particle physics community, where it is customary to include as coauthors tens or even hundreds of authors (Newman 2001). Clearly, there is a limit to the number of real acquaintances a given agent may manage.

We have also performed many simulations starting from different proportions of randomly distributed cooperators and defectors to investigate the effect of this parameter on the evolution of cooperation. In Figure 4 we show five different cases, the central image corresponding to the $50 \%$ situation. The images correspond to the lower left quarter of the right image in the middle row of Figure 3 with $\bar{k}=10, q=0.8$, $1<T<1.5$, and $0<P<0.5$.

Compared with the level of cooperation observed in simulations in static networks, we can say that results are consistently better for co-evolving networks. For example, the typical cases with $\bar{k}=10$ and $q=0.5$ and $q=0.8$ show significantly more cooperation than what was found in model and real social networks in previous work (Luthi et al. 2008). Even when there is a much lower rewiring frequency, i.e., with $q=0.2$, the cooperation levels are approximately as good as those observed in our previous study in which exactly the same RD scheme was used to update the agents' strategies, and the networks were of comparable size. The reason for this behavior is to be found in the added constraints imposed by the invariant network structure. The seemingly contradictory fact that an even higher cooperation level may be reached in static scale-free networks (Santos and Pacheco 1985) is theoretically interesting but easily dismissed as those graphs are unlikely models for social networks, which often show fat-tailed degree distribution functions but not pure power-laws (see, for instance, Amaral et al. 2000; Newman 2001). As a further indication of the latter, we shall see in the next section that indeed, emerging networks do not have a power-law degree distribution.

From the point of view of the evolutionary dynamics, it is interesting to point out that any given simulation run either ends up in full cooperation or full defection. When the full cooperation state of the population is attained, there is no way to switch back to defection by the intrinsic agent dynamics. In fact, all players are satisfied and have strong links with their cooperating neighbors. Even though a small amount of noise may still be present when deciding whether or not to rewire a link, since there are only cooperators around to imitate, there can be no strategy change and only very little link rewiring. On the other hand, well before this stable state is reached and there are still many defectors around, the system may experience some random drift that may drive it to full defection. The converse may also happen, but when the full defection state is reached, the situation is qualitatively different. In this case agents are unsatisfied, they will often try to rewire their links. However, all the other players around being also defectors, there will be constant changes of the local network structure. Thus the system will find itself in a fluctuating state, but this matters little for the bulk statistical properties of the population and of the network. To be assured that this is indeed the case, we have conducted some very long runs with all-defect end states. Global statistics do not change, except that the mean degree tends to increase slightly with time and the degree distribution function continues to evolve (see the next section). 

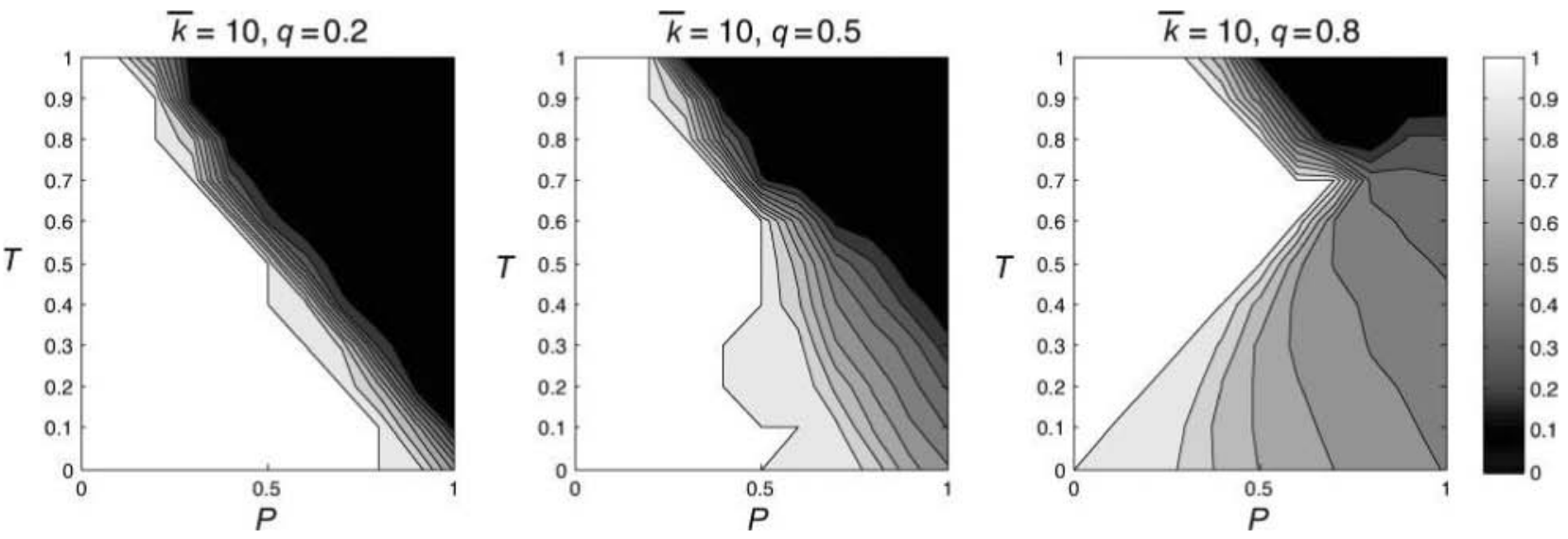

Figure 5 .

Cooperation level for the $\mathrm{SH}$ game.

Cooperation percentages as a function of the payoff matrix parameters for the SH game are shown in Figure 5 for $\bar{k}=10$ and $q=0.2,0.5$, and 0.8 . Note that in this case only the upper left triangle of the configuration space is meaningful. The $\mathrm{SH}$ is different from the PD since there are two evolutionarily stable strategies, which are therefore also NEs: one population state in which everybody defects and the opposite one in which everybody cooperates (see the previous section). Therefore, it is expected, and absolutely normal, that some runs will end up with all defect, while others will witness the emergence of full cooperation. In contrast, in the PD the only theoretically stable state is all-defect and cooperating states may emerge and be stable only by exploiting the graph structure and creating more favorable neighborhoods by breaking and forming ties. The value of the $\mathrm{SH}$ is in making manifest the tension that exists between the socially desirable state of full cooperation and the socially inferior but less risky state of defection (Skyrms 2004). The final outcome of a given simulation run depends on the size of the basin of attraction of either state, which is in turn a function of the relative values of the payoff matrix entries. To appreciate the usefulness of making and breaking ties in this game we can compare our results with what is prescribed by the standard RD solution. Referring to the payoff table of the previous section, let us assume that the column player plays $\mathrm{C}$ with probability $\alpha$ and $\mathrm{D}$ with probability $1-\alpha$. In this case, the expected payoffs of the row player are:

$$
E_{r}[C]=\alpha R+(1-\alpha) S
$$

and

$$
E_{r}[D]=\alpha T+(1-\alpha) P .
$$

Since the game is symmetric, the result for the column player is the same and $(\alpha \mathrm{C},(1-\alpha) \mathrm{D})$ is a NE in mixed strategies. We have numerically solved the equation for all the sam-

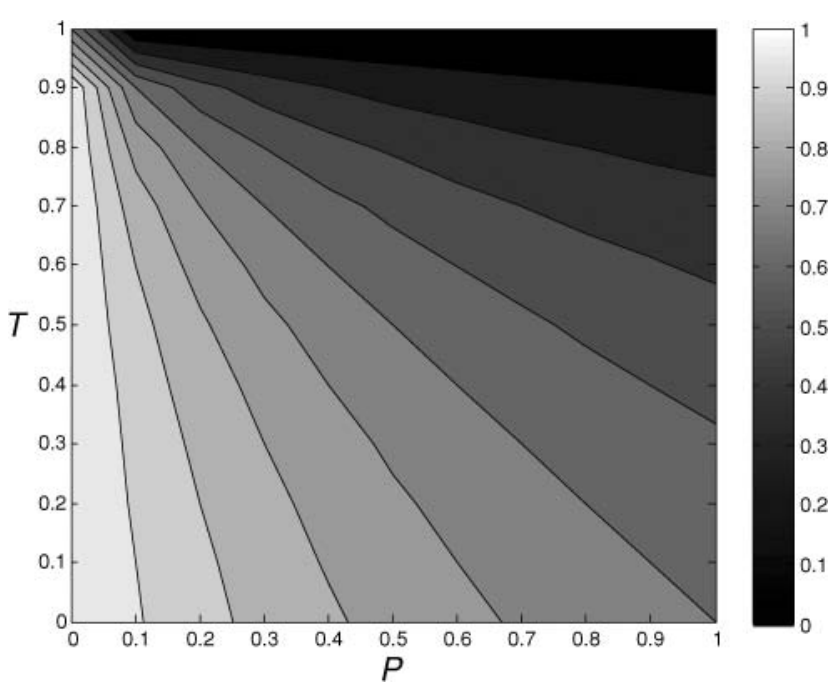

Figure 6.

Probabilities of cooperation for the mixed strategy NE as a function of the game's parameters for the Stag Hunt.

pled points in the game's parameter space, which gives the results shown in Figure 6. Let us now use the following payoff values in order to bring them within the explored game space (remember that NEs are invariant with respect to such a transformation; Weibull 1995):

\begin{tabular}{|l|l|l|}
\hline & $\mathrm{C}$ & $\mathrm{D}$ \\
\hline $\mathrm{C}$ & $(1,1)$ & $(0,2 / 3)$ \\
$\mathrm{D}$ & $(2 / 3,0)$ & $(1 / 3,1 / 3)$ \\
\hline
\end{tabular}

Substituting in (1) gives $\alpha=1 / 2$, i.e., the (unstable) polymorphic population should be composed by about half cooperators and half defectors. Now, if one looks at Figure 5 at the points where $P=1 / 3$ and $T=2 / 3$, one can see that this is approximately the case for the first image, within the limits of the approximations caused by the finite population size, the symmetry-breaking caused by the nonhomogeneous graph 

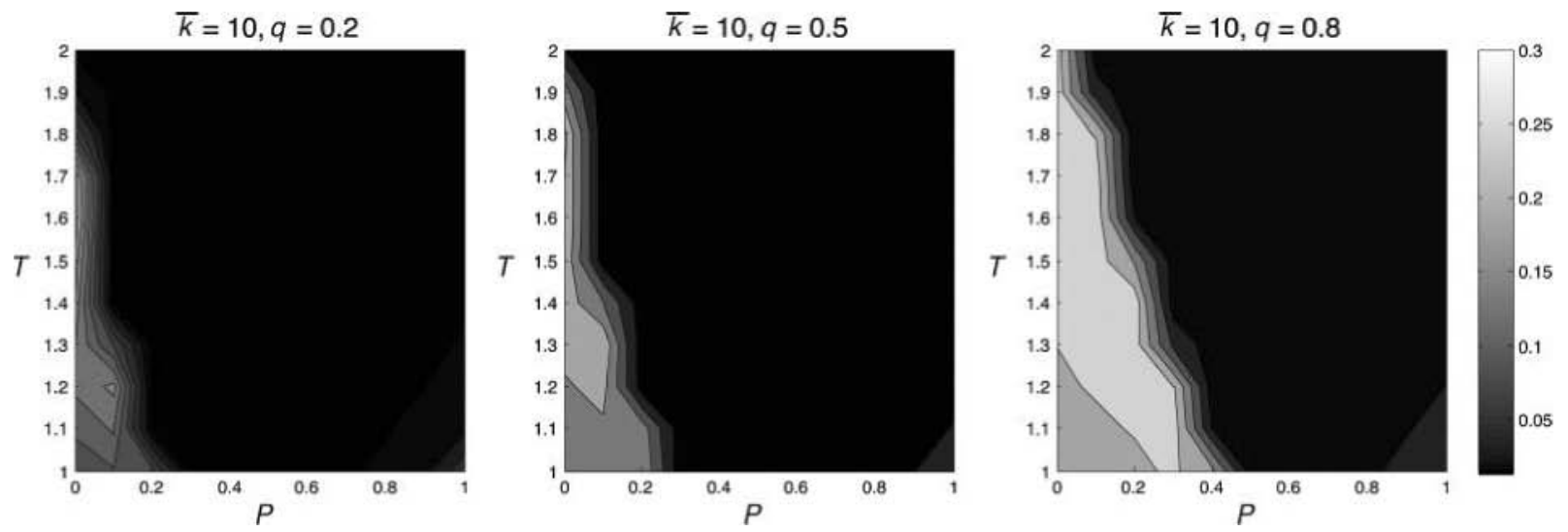

Figure 7.

Clustering coefficient level for the PD game. Lighter gray means more clustering.

structure, and the local nature of the RD. On the other hand, in the middle image and, to a greater extent, in the rightmost image, this point in the game space corresponds to pure cooperation. In other words, the nonhomogeneity of the network and an increased level of tie rewiring has allowed the cooperation basin to be enhanced with respect to the theoretical predictions of standard RD. Skyrms and Pemantle (2000) found the same qualitative result for very small populations of agents when both topology and strategy updates are allowed. It is reassuring that coordination on the payoff-dominant equilibrium can still be achieved in large populations as seen here.

\section{Structure of the Emerging Networks}

In this section we present a statistical analysis of the global and local properties of the networks that emerge when the pseudo-equilibrium states of the dynamics are attained. Let us start by considering the evolution of the average degree $\bar{k}$. Although there is nothing in our model to prevent a change in the initial mean degree, the steady-state average connectivity tends to increase only slightly. For example, in the PD with $q=0.8$ and $\bar{k}_{\text {init }}=5$ and $\bar{k}_{\text {init }}=10$, the average steady-state (ss) values are $\bar{k}_{\mathrm{ss}} \approx 7$ and $\bar{k}_{\mathrm{ss}} \approx 10.5$, respectively. Thus we see that, without imposing a constant $\bar{k}$ as in Zimmermann and Eguíluz (2005) and Santos et al. (2006), $\bar{k}$ nonetheless tends to increase only slightly, which nicely agrees with observations of real social networks (Barabási et al. 2002; Kossinets and Watts 2006; Tomassini and Luthi 2007). There is a special case when the steady state is all-defect and the simulation is allowed to run for a very long time $\left(2 \times 10^{4}\right.$ macrosteps $)$; in this case the link structure never really settles down, since players are unsatisfied, and $\bar{k}$ may reach a value of about 12 when starting with $\bar{k}=10$ and $q=0.8$.

Another important global network statistics is the average clustering coefficient $C$. The clustering coefficient $C_{i}$ of a node $i$ is defined as $C_{i}=2 E_{i} / k_{i}\left(k_{i}-1\right)$, where $E_{i}$ is the number of edges in the neighborhood of $i$. Thus $C_{i}$ measures the amount of "cliquishness" of the neighborhood of node $i$ and it characterizes the extent to which nodes adjacent to node $i$ are connected to each other. The clustering coefficient of the graph is simply the average over all nodes: $C=\frac{1}{N} \sum_{i=1}^{N} C_{i}$ (Newman 2003). Random graphs are locally homogeneous and for them $C$ is simply equal to the probability of having an edge between any pair of nodes independently. In contrast, real networks have local structures and thus higher values of $\mathrm{C}$. Figure 7 gives the average clustering coefficient $\bar{C}=\frac{1}{50} \sum_{i=1}^{50} C$ for each sampled point in the PD configuration space, where 50 is the number of network realizations used for each simulation. It is apparent that the networks self-organize and acquire local structure in the interesting, cooperative parts of the parameter space, since the clustering coefficients there are higher than that of the random graph with the same number of edges and nodes, which is $\bar{k} / N=10 / 1000=0.01$. Conversely, where defection predominates $C$ is smaller, witnessing of a lower amount of graph local restructuring. These impressions are confirmed by the study of the degree distribution functions (see below). The correlation between clustering and cooperation also holds through increasing values of $q$ : $C$ tends to increase from left to right in Figure 7 , a trend similar to that observed in the middle row of Figure 3 for cooperation. This correlation is maintained also for $\bar{k}=5$ and $\bar{k}=20$ (not shown).

As far as the clustering coefficient is concerned, the same qualitative phenomenon is observed for the SH, namely, the graph develops local structures and the more so the higher the value of $q$ for a given $\bar{k}$ (see Figure 8 ). Thus, it seems that evolution toward cooperation and coordination passes through a rearrangement of the neighborhood of the agents with respect to the homogeneous random initial situation, something that is made possible through the higher probability given to neighbors when rewiring a link, a stylized manifestation of the commonly occurring social choice of partners.

The degree distribution function (DDF) $p(k)$ of a graph represents the probability that a randomly chosen node has degree $k$ (Newman 2003). Random graphs are characterized by 

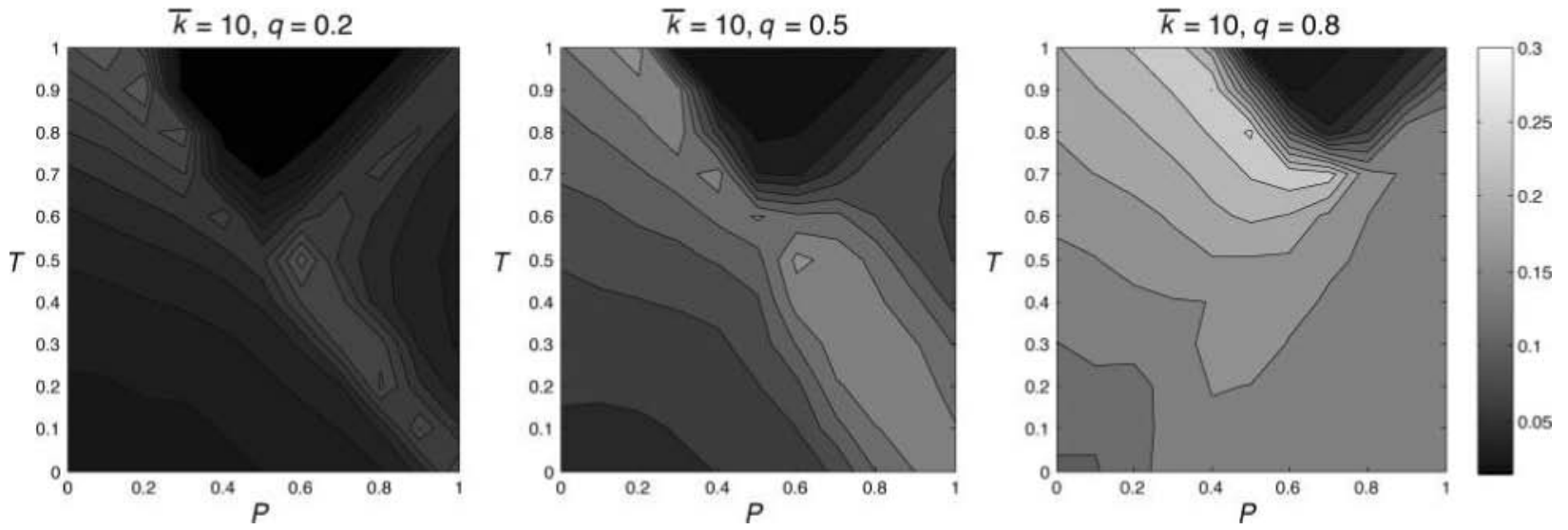

Figure 8.

Clustering coefficient level for the SH game.

DDF of Poissonian form, while social and technological real networks often show long tails to the right, i.e., there are nodes that have an unusually large number of neighbors (Newman 2003). In some extreme cases the DDF has a power-law form $p(k) \propto k^{-\gamma}$; the tail is particularly extended and there is no characteristic degree. The cumulative degree distribution function (CDDF) is just the probability that the degree is greater than or equal to $k$ and has the advantage of being less noisy for high degrees. Figure 9(a) shows the CDDFs for the PD for three cases of which two are in the cooperative region and the third falls in the defecting region (see Figure 3). The dotted curve refers to a region of the configuration space in which there is cooperation in the average but it is more difficult to reach, as the temptation parameter is high $(T=1.8, P=0.1)$. The curve has a rather long tail and is thus broad-scale in the sense that there is no typical degree for the agents. Therefore, in the corresponding network there are cooperators that are linked to many other cooperators. On the other hand, if one considers the dotted-dashed curve, which corresponds to a defecting region $(T=1.1, P=0.4)$, it is clear that the distribution is much closer to normal, with a well-defined typical value of the degree. Finally, the third thick curve, which corresponds to a region where cooperation is more easily attained $(T=1.1$, $P=0.1$ ), also shows a rather faster decay of the tail than the dotted line and a narrower scale for the degree. Nevertheless, it is right-skewed, indicating that the network is no longer a pure random graph. Since we use linear-log scales, the dotted curve has an approximately exponential or slower decay, given that a pure exponential would appear as a straight line in the plot. The tail of the thick curve decays faster than an exponential, while the dashed-dotted curve decays even faster. Almost the same observations also apply to the SH case, shown in Figure 9(b). These are quite typical behaviors and we can conclude that, when cooperation is more difficult to reach, agents must better exploit the link-redirection degree of freedom in order for cooperators to stick together in sufficient quantities and protect themselves from exploiting defectors during the co-evolution.
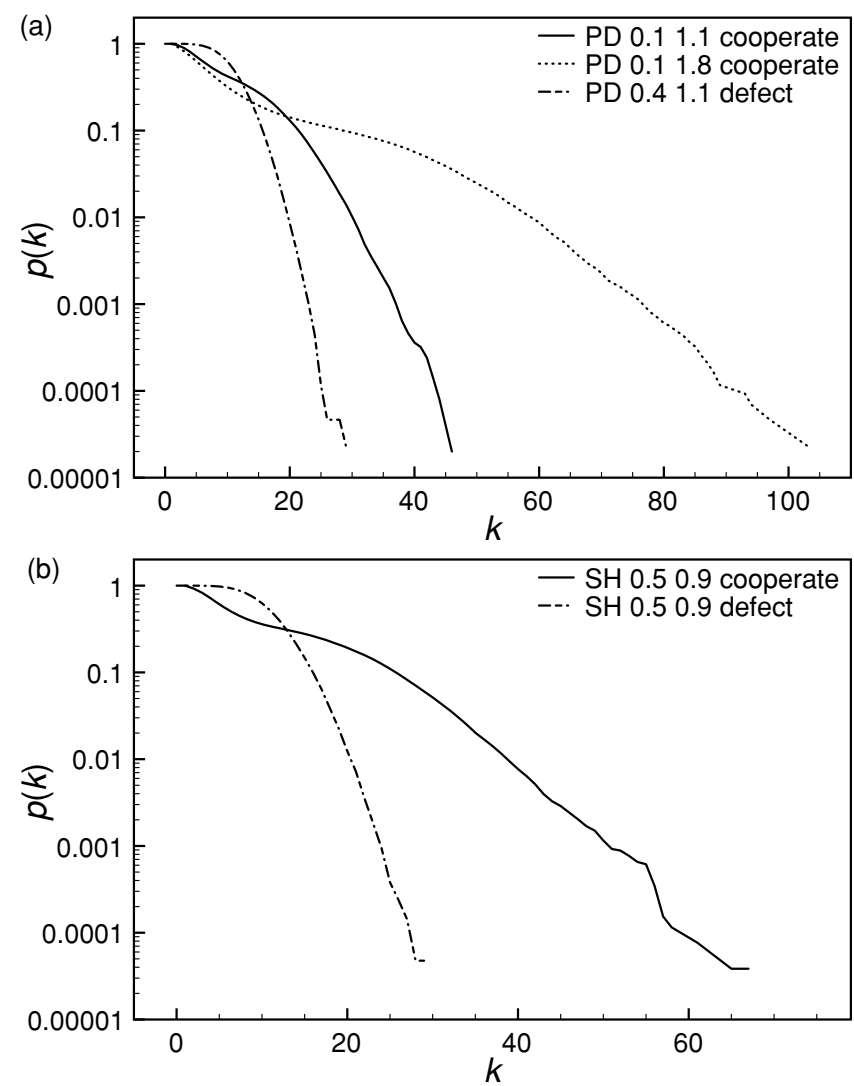

Figure 9

Cumulative degree distributions. Average values over 50 runs. (a): PD, (b): $\mathrm{SH}$. $q=0.8, \bar{k}=10$. Linear-log scales

When the situation is either more favorable for cooperation, or defection easily prevails, network rearrangement is less radical. In the limit of long simulation times, the defection case leads to networks that have degree distribution close to Poissonian and are thus almost random. Figure 10 shows such a case for the PD. The dashed curve is the CDDF at some intermediate time, when full defection has just been reached but the network is still strongly reorganizing itself. Clearly, the distribution has a long tail. However, if the simulation is 


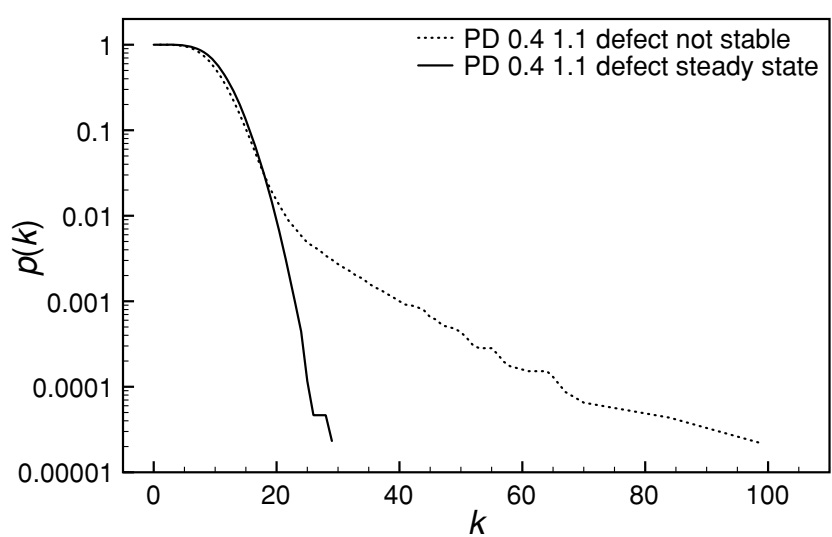

Figure 10.

Cumulative degree distributions for the PD in case of defection before (dotted line) and after (thick line) reaching a steady state. Linear-log scales.

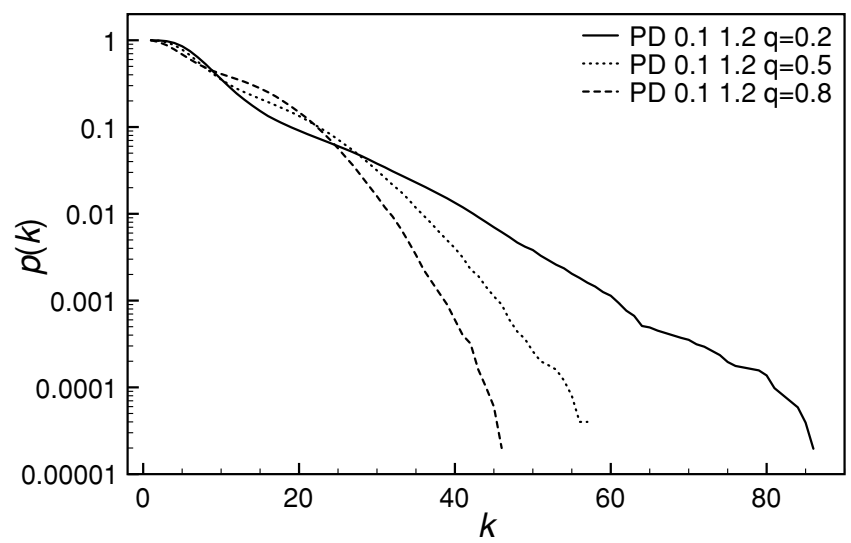

Figure 11.

Cumulative degree distribution functions for three values of $q$, for the same point in the PD configuration space in the cooperating region.

continued until the topology is quite stable at the mesoscopic level, the distribution becomes close to normal (thick curve).

Finally, it is interesting to observe the influence of the $q$ parameter on the shape of the degree distribution functions for cooperating networks. Figure 11 reports average curves for three values of $q$. For high $q$, the cooperating steady state is reached faster, which gives the network less time to rearrange its links. For lower values of $q$ the distributions become broader, despite the fact that rewiring occurs less often, because cooperation in this region is harder to attain and more simulation time is needed.

Influence of timing Figure 12 depicts a particular cut in the configuration space as a function of the synchronicity parameter $f$. The main remark is that asynchronous updates give similar results, in spite of the difference in the number of agents that are activated in a single microstep. In contrast, fully synchronous update $(f=1)$ appears to lead to a slightly less favorable situation for cooperation. Since fully synchronous update is physically unrealistic and can give spurious results

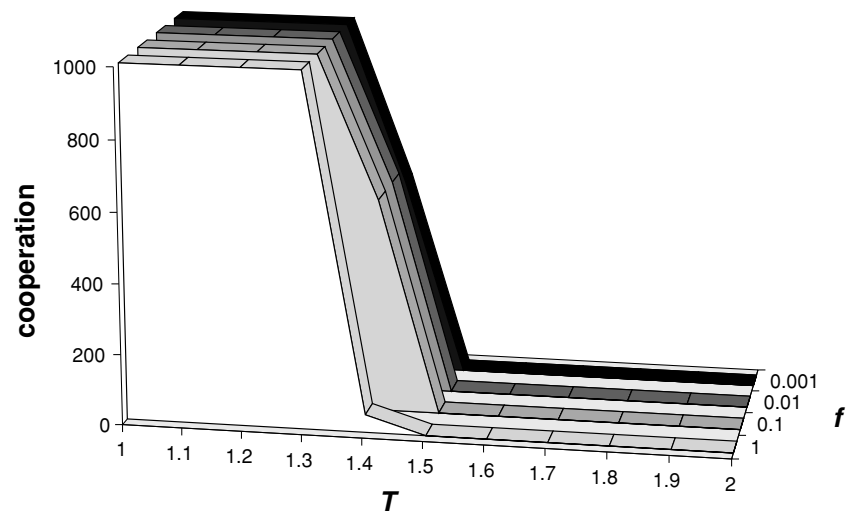

Figure 12.

Cooperation levels in the PD for $P=0.1$ and $1 \leq T \leq 2$ as a function of the synchronicity parameter $f$.

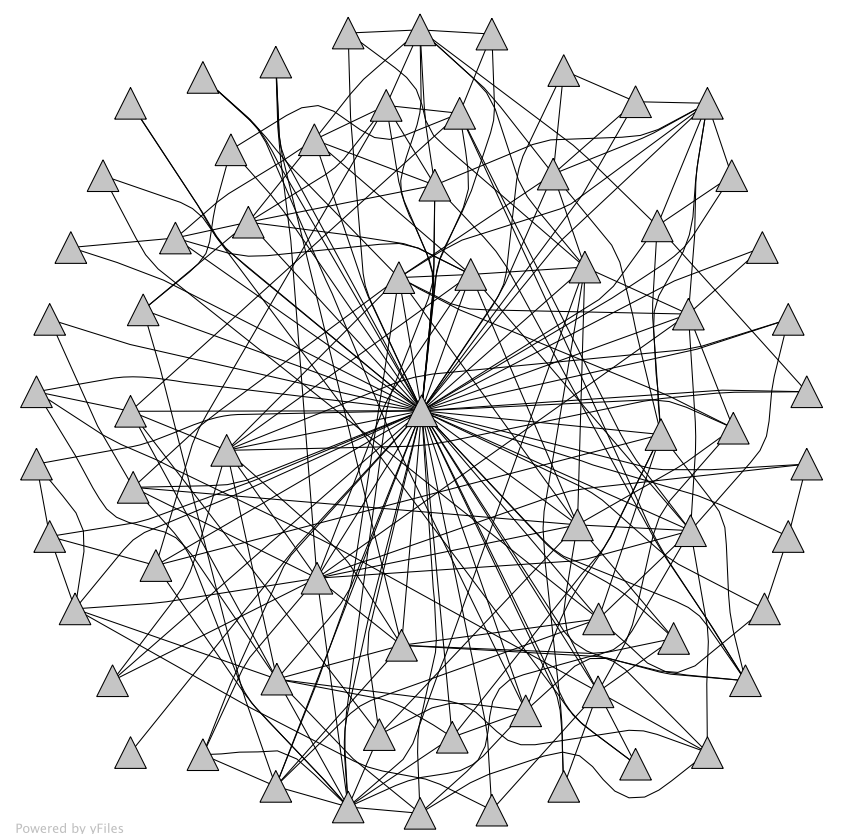

Figure 13.

Example of a tightly packed cluster of cooperators for PD networks. $T=1.8$, $P=0.1$, and $q=0.8$.

due to symmetry, we suggest using fully or partially asynchronous update for agent's simulation of artificial societies.

\section{Clusters}

We have seen in the previous section that, when cooperation is attained in both games as a quasi-equilibrium state, the system remains stable through the formation of clusters of players using the same strategy. In Figure 13 one such typical cluster corresponding to a situation in which global cooperation has been reached in the PD is shown. Although all links toward the "exterior" have been suppressed for clarity, one can clearly see that the central cooperator is a highly connected node and there are many links also between the other neighbors. Such a tightly packed structure has emerged to protect cooperators 

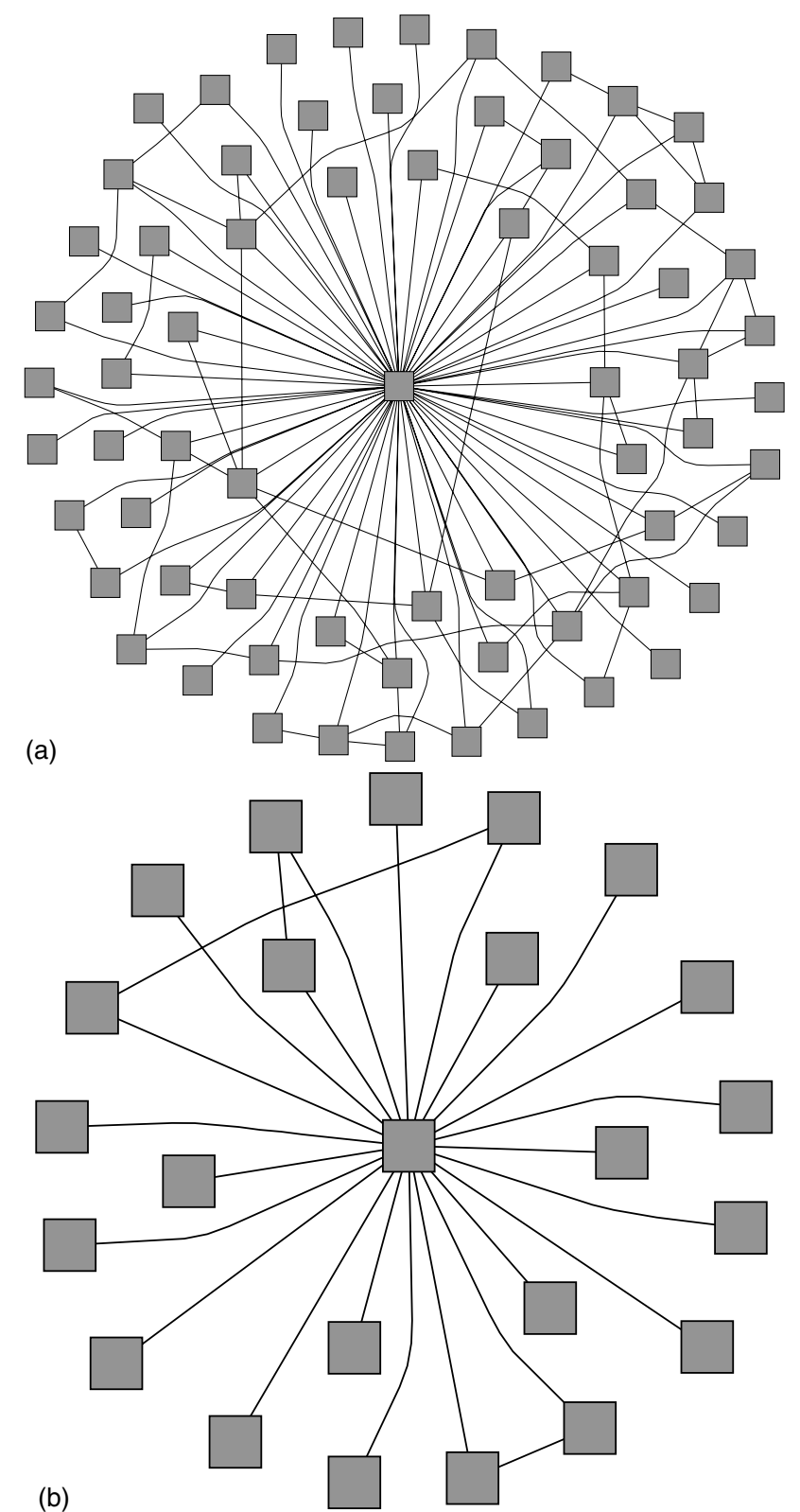

Figure 14.

Example of defector clusters for PD networks, for $T=1.8, P=0.3$, and $q=0.8$. Clusters like (a) exist only just after the all-defect state is reached. When a steady state is reached only clusters like (b) are present in a network of defectors.

from defectors that, at earlier times, were trying to link to cooperators to exploit them. These observations explain why the degree distributions are long-tailed (see previous section), and also the higher values of the clustering coefficient in this case (see section "Structure of the Emerging Networks").

When the history of the stochastic process is such that defection prevails in the end, the situation is totally different. Figure 14(a) and 14(b) show two typical examples of cluster structures found during a simulation. Figure 14(a) refers to a stage in which the society is composed solely by defectors. However, the forces of the links between them are low, and so many defectors try to dismiss some of their links. This situation lasts for a long simulated time (actually, the system is never at rest, as far as the links are concerned) but the dense clusters tend to dissolve, giving rise to structures such as the one shown in Figure 14(b). If one looks at the degree distribution at this stage (Figure 10) it is easy to see that the whole population graph tends to become random.

The SH case is very similar, which is a relatively surprising result. In fact, when cooperation finally takes over in regions of the configuration space where defection would have been an almost equally, likely final state, players are highly clustered and there are many highly connected individuals, while in less conflicting situations the clusters are less dense and the degree distribution shows a faster decay of the tail. On the other hand, when defection is the final quasi-stable state, the population graphs lose a large part of their structure. Thus, the same topological mechanisms seem to be responsible for the emergence of cooperation in the PD and in the SH. The only previous study that investigates the structure of the resulting networks in a dynamical setting is, to our knowledge, Zimmermann and Eguíluz (2005), where only the PD is studied. It is difficult to meaningfully compare our results with theirs, as their model differs from ours in many ways. Thus they use a deterministic hard-limit rule for strategy update which is less smooth than our stochastic local RD. Moreover, they study the PD in a reduced configuration space, only links between defectors can be broken, and links are rewired at random. They concentrate on the study of the stability of the cooperating steady states against perturbations, but do not describe the topological structures of the pseudo-equilibrium states in detail. Nevertheless, it is worthy to note that the degree distribution functions for cooperators and defectors follow qualitatively the same trend, i.e., cooperator networks have distributions with fatter tails to the right than defector networks.

\section{Conclusions and Future Work}

Using two well-known games that represent conflicting decision situations commonly found in animal and human societies, we have studied by computer simulation the role of the dynamically networked society's structure in the establishment of global cooperative and coordinated behaviors, which are desirable outcomes for the society's welfare. Starting from randomly connected players which only interact locally in a restricted neighborhood, and allowing agents to probabilistically and bilaterally dismiss unprofitable relations and create new ones, the stochastic dynamics lead to pseudo-equilibria of either cooperating or defecting agents. With respect to standard RD results for mixing populations, we find that there is a sizeable configuration space region in which cooperation may emerge and be stable for the PD, whereas the classical result predicts total defection. For the 
$\mathrm{SH}$, where both all-cooperate and all-defect steady states are theoretically possible, we show that the basin of attraction for cooperation is enhanced. Thus, the possibility of dismissing a relationship and creating a new one does indeed increase the potential for cooperation and coordination in our artificial society. The self-organizing mechanism consists in both games in forming dense clusters of cooperators which are more difficult to dissolve by exploiting defectors. While the beneficial effect of relational or geographical static population structures on cooperation was already known from previous studies, here we have shown that more realistic dynamic social networks may also allow cooperation to thrive. Future work will deal with the stability of the cooperating states against stronger perturbations than merely the implicit noise of the stochastic dynamics. We also intend to study more fully the structure of the emerging clusters and their relationships, and we plan to extend the model to other important paradigmatic games such as Hawks-Doves and coordination games.

\section{Acknowledgments}

E. Pestelacci and M. Tomassini are grateful to the Swiss National Science Foundation for financial support under contract number 200021-111816/1. We thank the anonymous reviewers for useful remarks and suggestions.

\section{Note}

1. A further refinement of the concept could take obsolescence phenomena into account. For instance, in the same way that pheromone trails laid down by ants evaporate with time, we could introduce a progressive loss of strength of the links proportional to the time during which there is no interaction between the concerned agents. For the sake of simplicity, we prefer to stick with the basic model in this article.

\section{References}

Abramson G, Kuperman M (2001) Social games in a social network. Physics Reviews E 63: 030901.

Amaral LAN, Scala A, Barthélemy M, Stanley HE (2000) Classes of smallworld networks. Proceedings of the National Academy of Sciences USA 97: 11149-11152.

Axelrod R (1984) The Evolution of Cooperation. New York: Basic Books.

Barabási A-L, Jeong H, Néda Z, Ravasz E, Schubert A, Vicsek T (2002) Evolution of the social network of scientific collaborations. Physica A 311: 590-614.

Bollobás B (1998) Modern Graph Theory. Berlin: Springer.

Hauert C, Doebeli M (2004) Spatial structure often inhibits the evolution of cooperation in the snowdrift game. Nature 428: 643-646.
Hebb DO (1949) The Organization of Behavior. New York: Wiley.

Hofbauer J, Sigmund K (1998) Evolutionary Games and Population Dynamics. Cambridge: Cambridge University Press.

Huberman BA, Glance NS (1993) Evolutionary games and computer simulations. Proceedings of the National Academy of Sciences USA 90: 7716-7718.

Kossinets G, Watts DJ (2006) Empirical analysis of an evolving social network. Science 311: 88-90.

Luthi L, Giacobini M, Tomassini M (2006) A minimal information prisoner's dilemma on evolving networks. In: Artificial Life X (Rocha LM, ed), 438-444. Cambridge, MA: MIT Press.

Luthi L, Pestelacci E, Tomassini M (2008) Cooperation and community structure in social networks. Physica A 387: 955-966.

Myerson RB (1991) Game Theory: Analysis of Conflict. Cambridge, MA: Harvard University Press.

Newman MEJ (2001) Scientific collaboration networks. I. Network construction and fundamental results. Physics Review E 64: 016131.

Newman MEJ (2003) The structure and function of complex networks. SIAM Review 45: 167-256.

Nowak MA, May RM (1992) Evolutionary games and spatial chaos. Nature 359: 826-829.

Nowak MA, Sigmund K (2000) Games on grids. In: The Geometry of Ecological Interactions: Simplifying Spatial Complexity (Dieckmann U, Law R, Metz JAJ, eds), 135-150. Cambridge: Cambridge University Press.

Santos FC, Pacheco JM (2005) Scale-free networks provide a unifying framework for the emergence of cooperation. Physical Review Letters 95: 098104.

Santos FC, Pacheco JM, Lenaerts T (2006) Cooperation prevails when individuals adjust their social ties. PLoS Computational Biology 2: 12841291.

Skyrms B (2004) The Stag Hunt and the Evolution of Social Structure. Cambridge: Cambridge University Press.

Skyrms B, Pemantle R (2000) A dynamic model for social network formation. Proceedings of the National Academy of Sciences USA 97: 93409346.

Szabó G, Fáth G (2007) Evolutionary games on graphs. Physics Reports 446: 97-216.

Tomassini M, Luthi L (2007) Empirical analysis of the evolution of a scientific collaboration network. Physica A 385: 750-764.

Tomassini M, Luthi L, Giacobini M (2006) Hawks and doves on small-world networks. Physics Reviews E 73: 016132.

Tomassini M, Luthi L, Giacobini M, Langdon WB (2007) The structure of the genetic programming collaboration network. Genetic Programming and Evolvable Machines 8: 97-103.

Wang S, Szalay MS, Zhang C, Csermely P (2008) Learning and innovative elements of strategy adoption rules expand cooperative network topologies. PLoS ONE 3(4): e1917. doi:10.1371/journal.pone.0001917

Weibull JW (1995) Evolutionary Game Theory. Cambridge, MA: MIT Press.

Zimmermann MG, Eguíluz VM (2005) Cooperation, social networks, and the emergence of leadership in a prisoner's dilemma with adaptive local interactions. Physics Reviews E 72: 056118. 